\title{
ISOLATION AND CHARACTERIZATION OF A POLLEN- SPECIFIC GENE ZMSTK2_USP FROM ZEA MAYS
}

\author{
JAVEED, A. ${ }^{1 \Psi}$ - FAN, M. X. ${ }^{1 \Psi}-$ SHI, L. ${ }^{2}-$ ZHANG, C. Y. ${ }^{1}-$ MA, W. J. ${ }^{1}-$ CHEN, M. M. ${ }^{1}-$

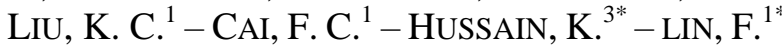 \\ ${ }^{1}$ College of Bioscience and Biotechnology, Shenyang Agricultural University \\ No. 120 Dongling Road, Shenyang, 110866 Liaoning, China \\ (phone: +86-24-8848-7163 \\ ${ }^{2}$ Corn Research Institute, Liaoning Academy of Agricultural Sciences \\ Shenyang, Liaoning, China \\ ${ }^{3}$ Department of Botany, University of Gujrat HH Campus \\ Gujrat, Pakistan \\ ${ }^{\Psi}$ These authors have equal contribution to this paper. \\ *Corresponding authors \\ e-mail: fenglinsn@126.com; Khalid.hussain@uog.edu.pk \\ (Received $25^{\text {th }}$ Aug 2017; accepted $8^{\text {th }}$ Nov 2017)
}

\begin{abstract}
Microsporogenesis in flowering plants is a complex process involving a range of gene expression. In the present study, a pollen-specific gene ZmSTK2_USP (serine threonine kinase) was isolated from Zea mays. Mutant ZmSTK2_USP showed 56.7\% pollen viability capacity, $48.92 \%$ germination rates comparing with wild-type. Northern Blot results showed that ZmSTK2_USP were specifically expressed in mature pollen. The expression level was highest at late pollen development, followed by bicellular stage. And at early pollen stage, no expression was detected. GUS activity was detected in middle stage, late stage of pollens and germinated pollen. These results indicated that ZmSTK2_USP expressed at late pollen development and was pollen-specific.
\end{abstract}

Keywords: maize, serine/threonine kinase, pollen development, mutant, expression pattern, GUS activity

\section{Introduction}

The floral organ development of plants needs to cooperate with the expression and regulation of various floral organ-specific genes, including a large number of genes related to anther or pollen development.

In plants, a number of anther- or pollen-specific genes have been isolated and identified. Hanson et al. (1989) reported that Zmcl3 expressed in maize pollen and pollen tube. OSIPA promoter conferred pollen-specific expression in both rice and Arabidopsissystems (Swapna et al., 2011). TaPSG719 isolated from wheat were specific for mature pollen, and undetectable in other tissues (Jin and Bian, 2004). TUA1 expressed in stamens and mature pollen in Arabidopsis thaliana development (Carpenter et al., 1992). SBgLR promoter in potato was identified as critical for gene specific expression in pollen grains (Zhou et al., 2010).

In the present study, we reported a pollen-specific gene ZmSTK2_USP (serine threonine kinase) isolated from Zea mays. Mutant ZmSTK2_USP pollen viability decreased, causing pollen germination disorder and loss of competitiveness, thus affecting the fertility of maize. 


\section{Materials and methods}

\section{Plant materials and growth conditions}

Table 1 lists all the primers used in this study. Mu-induced mutant ZmSTK2_USP was donated by Dr. Dooner (Rutgers University, USA) and the insertion information were previously published by Fu et al. (2001). The wild type $\mathrm{McC}$, transgenic lines and mutants were grown in greenhouse with $16 \mathrm{~h}$ light $/ 8 \mathrm{~h}$ dark cycle.

Table 1. The list of primers used in the present study

\begin{tabular}{c|c|c}
\hline Gene & Forward primer & Reverse primer \\
\hline $\begin{array}{c}\text { Mu insertion lines } \\
M u-F \\
\text { ZmSTK2_USPA }\end{array}$ & $\begin{array}{c}\text { TGTGGTTGTCTTGAACCGCT } \\
(\text { SO016 })\end{array}$ & $\begin{array}{c}\text { GCCTCYATTTCGTCGAATCC } \\
\text { AAGCACAATGGGCAGGTACA } \\
(\text { S2011 })\end{array}$ \\
\hline $\begin{array}{c}\text { Cloning } \\
\text { ZmSTK2_USP } B\end{array}$ & ATGGGCAGGTACAGCGACG & GTTGTAGTTGGACCTCCATGGA \\
\hline $\begin{array}{c}\text { Control genes } \\
\text { Actin }\end{array}$ & GTTGGGCGTCCTCGTCA & TGGGTCATCTTCTCCCTGTT \\
\hline $\begin{array}{c}\text { Southern Blot Analysis } \\
\text { ZmSTK2_USP } E\end{array}$ & AGACGGGCATGCTGGGGGTGAAGT & ATGGCGGCGACGGGCTGGTGT \\
\hline $\begin{array}{c}\text { GUS for transgenic } \\
\text { lines }\end{array}$ & GCAACTGGACAAGGCACT & GAGCGTCGCAGAACATTACA \\
\hline
\end{tabular}

\section{In vitro pollen viability and germination assays}

The pollen of wild type McC and mutant zmstk2_usp was collected at 10:00 a.m. every day when the tassel began to loose pollen. After mixing, some of the pollen was quickly brought back to the laboratory within an ice pack.

Proper amount of pollen was put onto clean slides, 2 drops of I2-KI solution were dropped. After treatment with $10 \mathrm{~min}$ at 30 constant temperature, the pigmentation of pollen grains were observed under the microscope (ZEISS Axio Scope. A1) and photographed. Mature pollen grains are dark colored, indicating that pollen has strong vitality and shallow coloration indicated that pollen activity is poor or not vigorous. Pollen grains, total no less than 100, were observed under 5 of visual field. Count the number of dark and light colors of pollen grains, and calculate the pollen viability capacity $(\%)$. Values are mean with $n=3$.

Pollen germination experiments of wild type $\mathrm{McC}$ and mutant zmstk2_usp were carried out in vitro referring to the methods described in Schreiber and Dresselhaus (2003). A small amount of pollen were immediately and uniformly scattered to the slides coated with the pollen germination medium [medium formula is followed according to Wang et al. (2017), incubated $2 \mathrm{~h}$ in a petri dish, with wet filter paper, at constant temperature illumination and observed under the microscope (ZEISS Axio Scope. A1) within 5 of visual field and photographed. The number of germinated and non-germinated pollen grains was counted and the pollen germination rates $(\%)$ were calculated. Values are mean with $\mathrm{n}=3$. 


\section{Construction of the ZmSTK2_USP expression vector, and maize transformation}

The upstream primer STK2B-F and downstream primer STK2B-R were designed based on the sequence of the ZmSTK2_USP gene and listed in Table 1. STK2B-F with BamHI and STK2B-R with HindIII cleavage site were employed used to amplifiy coding sequences of ZmSTK2_USP using McC pollen cDNA as template. ZmSTK2_USP target fragments were introduced into the TA clone plasmid, sequenced to gent positive cloning. The plasmids with positive cloning were digested by BamHI and HindIII. ZmSTK2_USP target fragments were cloned into pCAMBIA1301 between cleavage sites of BamHI and HindIII, which an expression vector containing a ZmSTK2_USP-GUS fusion gene was constructed.

\section{Southern blot analysis}

Leaf DNA of T1 transgenic plants with pCAMBIA1301-GUS was isolated and amplified using primers GUS-F and GUS-R listed in Table 1. The DNA probe was labeled using DIG. Genome DNA $(40 \mu \mathrm{g})$ of positive T1 transgenic plants was digested using Hind III, electrophoresised, then transferred to membrane, hybridized and detected according to DIG High Prime DNA Labeling and Detection Starter Kit I (Roche). The colored membrane was recorded by digital camera.

\section{Histochemical GUS staining}

For GUS activity analysis, we regularly collected various tissues at different developmental stages (organs) of $\mathrm{McC}$ and transgenic $\mathrm{T}_{2}$ generation of ZmSTK2_USPGUS, including immature male flowers, mature male flowers, and mature anthers, three different developmental stages (early stage of pollen development, middle stage of pollen development and late stage of pollen development) and pollen tube.

According to the method described by Jefferson et al. (1987), we put various tissues (organs) into $90 \%$ acetone stationary liquid for $20 \mathrm{~min}$ in $-20{ }^{\circ} \mathrm{C}$ refrigerator, PBS buffer rinses 2 times using PBS buffer, each time $10 \mathrm{~min}$, then added GUS staining solution X-Gluc (TritonX-100, 20\% methanol), incubated $16 \mathrm{~h}$ at $37{ }^{\circ} \mathrm{C}$ in dark. After that, the green tissues (organs) was decolorized 2 times using 70\%-100\%-70\% (v/v) ethanol, observed under microscope (ZEISS Axio Scope. A1) and photograph.

\section{Results and discussion}

\section{Phenotypic analysis of ZmSTK2_USP mutants}

$M u$-induced ZmSTK2_USP, a non-autonomous $1.4 \mathrm{~kb} \mathrm{Mu}$ element at the beginning of the $5^{\text {th }}$ exon of ZmSTK2_USP (Fig. 1A), was obtained from Rutgers University, USA (Dooner, unpublished). ZmSTK2_USP mutants were detected using the primers listed in Table 1. 


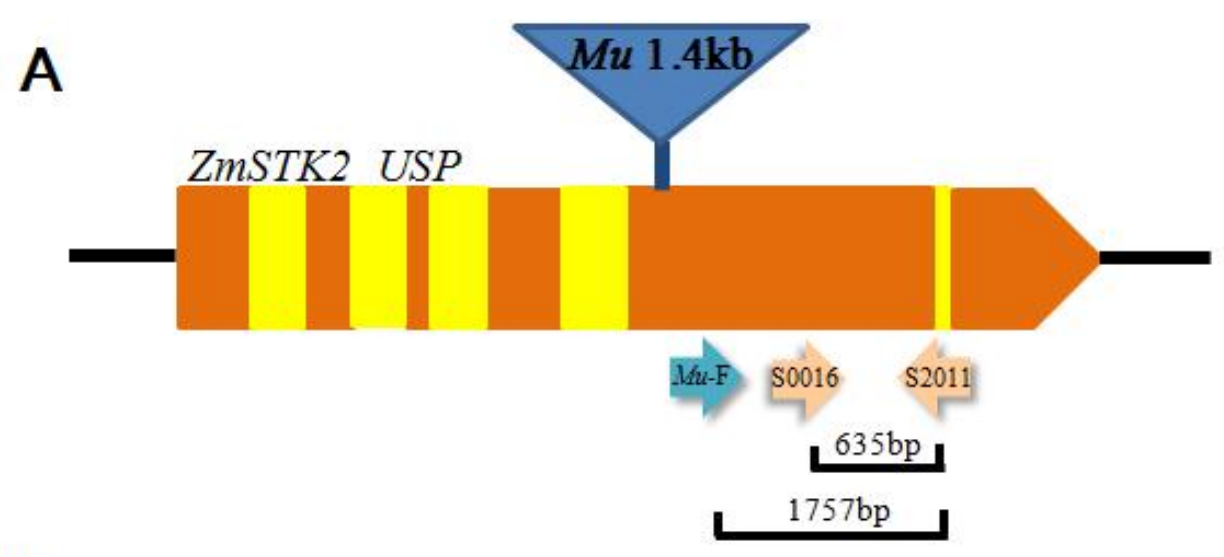

B

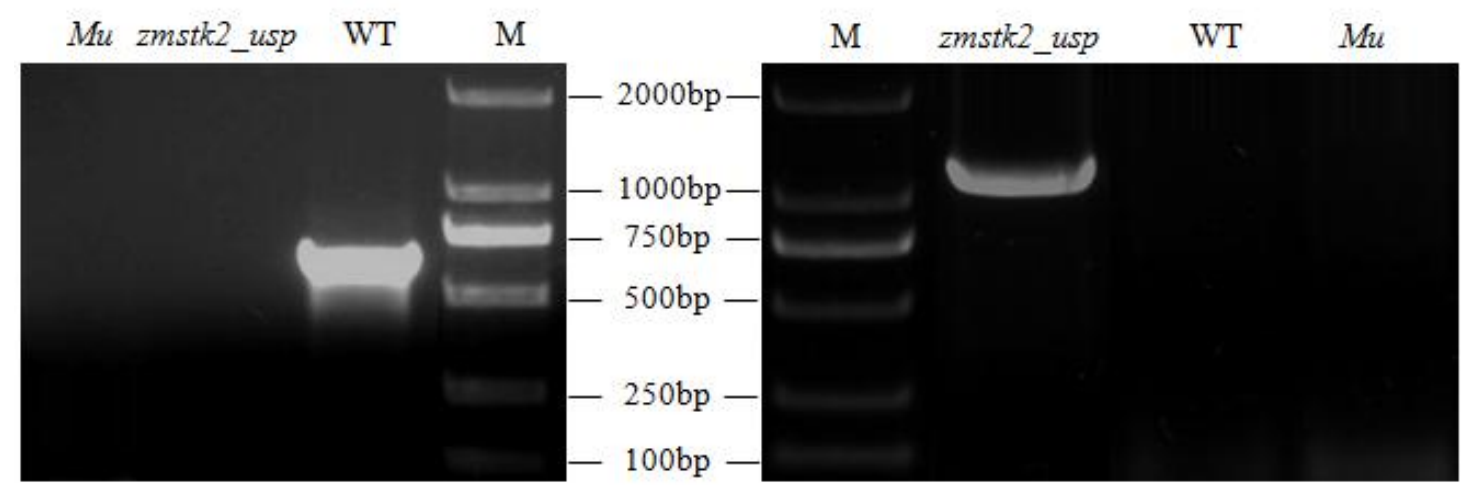

Figure 1. Mu insertion mutation and detection of homozygous mutant. Note: A. Schematic view of the genomic structure of ZmSTK2_USP in maize and sites of Mu insertions; B. RT-PCR test results. $M$ denotes DL2000. Mu was used as a negative control. The orange solid rectangle is the exon; the yellow solid rectangle is the intron; the inverted blue solid triangle is the Mu transposon and its inserted site (the fifth exon origin); the blue arrow is Mu primers and directions; the pink arrows are a pair of primers and directions on ZmSTK2_USP gene; and the black line segment is the length of the RT-PCR product corresponding to the primer combination

Fig. 2 shows phenotypic traits of wild-type $\mathrm{McC}$ and mutant ZmSTK2_USP during the whole period of growth and development. Both wild-type and mutant were semi-compact plant type (Figs. 2D, $d$ and E,e) with 12-15 tassel branch (Figs. $2 E$ and $e$ ), purple anther (Figs. $2 F$ and $f$ ) and purple durum grain (Figs. $2 G$ and $g$ ). In our previous study, ZmSTK2_USP belonged to receptor-like cytoplasmic kinase (RLCK) and was pollen-specific (Wang et al., 2014; Zhou et al., 2014; Wang et al., 2017). Hence, we observed pollen grains of wild-type plants and mutants. The pollen grains of wild type $\mathrm{McC}$ are dyed darkly by $\mathrm{I}_{2}$-KI , very plump and most of them were germinated, while most of mutant pollen grains are partly wrinkled and hollow, dyed brown and not germinated (Figs. 2A, $a$ and B,b). The mutant ZmSTK2_USP was artificially pollinated with wild type McC plants, and dyed with aniline blue after $6 \mathrm{~h}$, pollen grain germination and pollen tube elongation of ZmSTK2_USP were very slow and half of them did not germinated comparing with wild-type (Figs. $2 C$ and $c$ ). 


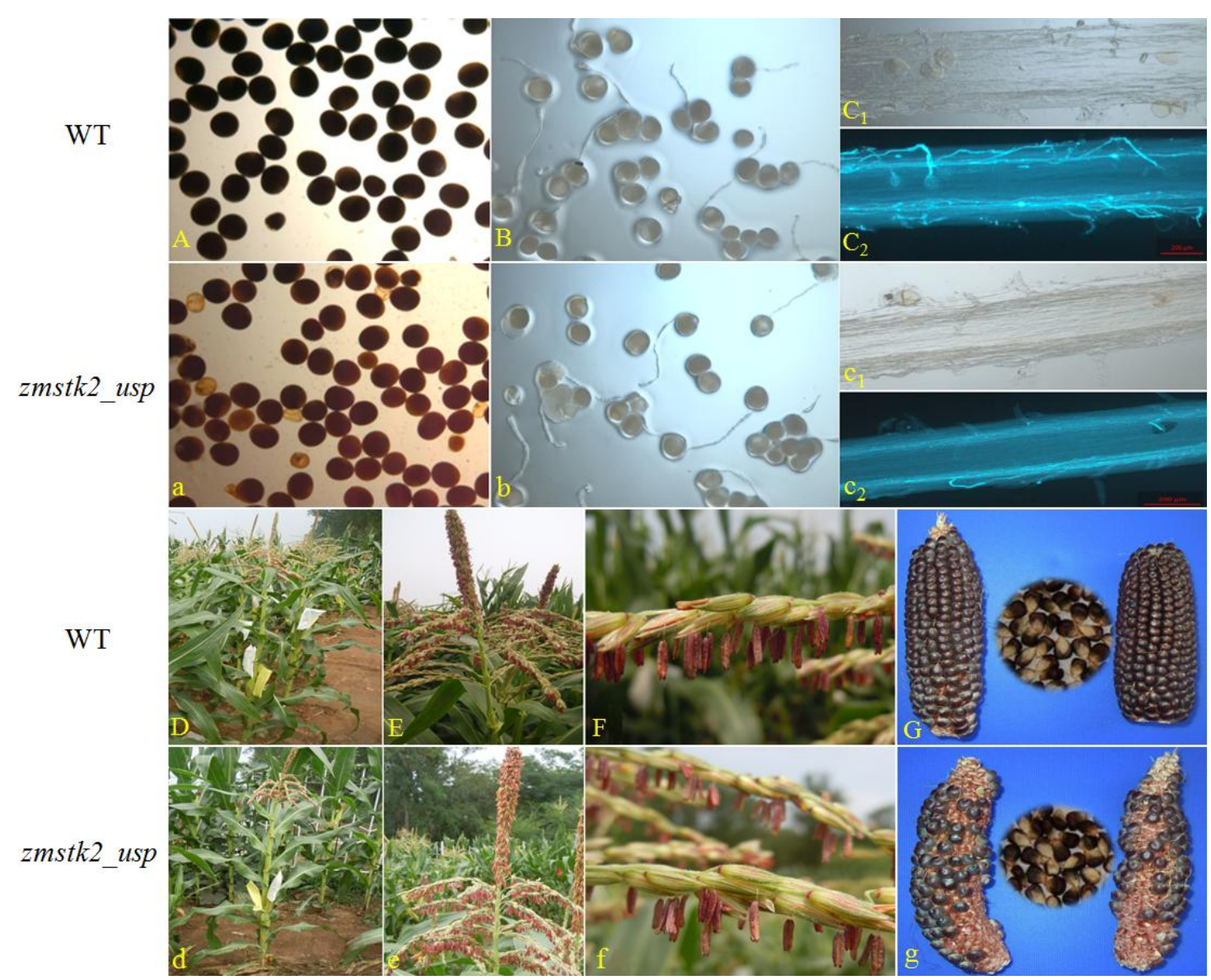

Figure 2. Phenotypic comparison of wild-type and mutant zmstk2_usp. Note: A and a. Images of pollen viability obtained after staining with $I_{2}-K I ; B$ and $b$. Pollen germination in vitro after $2 \mathrm{~h} ; \mathrm{C}_{1}, C_{2}, c_{1}$ and $c_{2}$. In vivo of germinated pollen grains in silks after $6 \mathrm{~h} ; \mathrm{Cl}$ and cl were observed in bright field; $C 2$ and c2 were observed under fluorescence; $D$ and d. Plant type of the wild type and mutant; $E$ and $e$ Tassel of the wild type and mutant; $F$ and $f$. Anther of the

wild type and mutant; $G$ and $g$. Grain color and grain type of the wild type and mutant

Fig. 3 shows the statistical analysis results of pollen viability capacity, germination rates, filled grains per spike of mutant ZmSTK2_USP and wild-type plants. Comparing with wild-type, mutants showed extremely significant difference with $56.7 \%$ pollen viability capacity, $48.92 \%$ germination rates. The filled grains per spike of mutant ZmSTK2_USP were around 40\% of wild-type. In summary, $M u$-induced ZmSTK2_USP cause decreased pollen viability, disordered pollen germination, and lower competitiveness, thus affected the fertility of maize.

\section{ZmSTK2_USP expression pattern}

To clarify the expression profiles of ZmSTK2_USP gene in Maize, we performed Northern Blot and qRT-PCR analysis (Fig. 4). Northern Blot results showed that ZmSTK2_USP were specifically expressed in mature pollen and no expression was found in other tissues (organs). The expression level was highest at late pollen development, followed by bicellular stage. And at early pollen stage, no expression was detected. The pattern of ZmSTK2_USP expression indicates that ZmSTK2_USP expressed at late pollen development and was pollen-specific. 


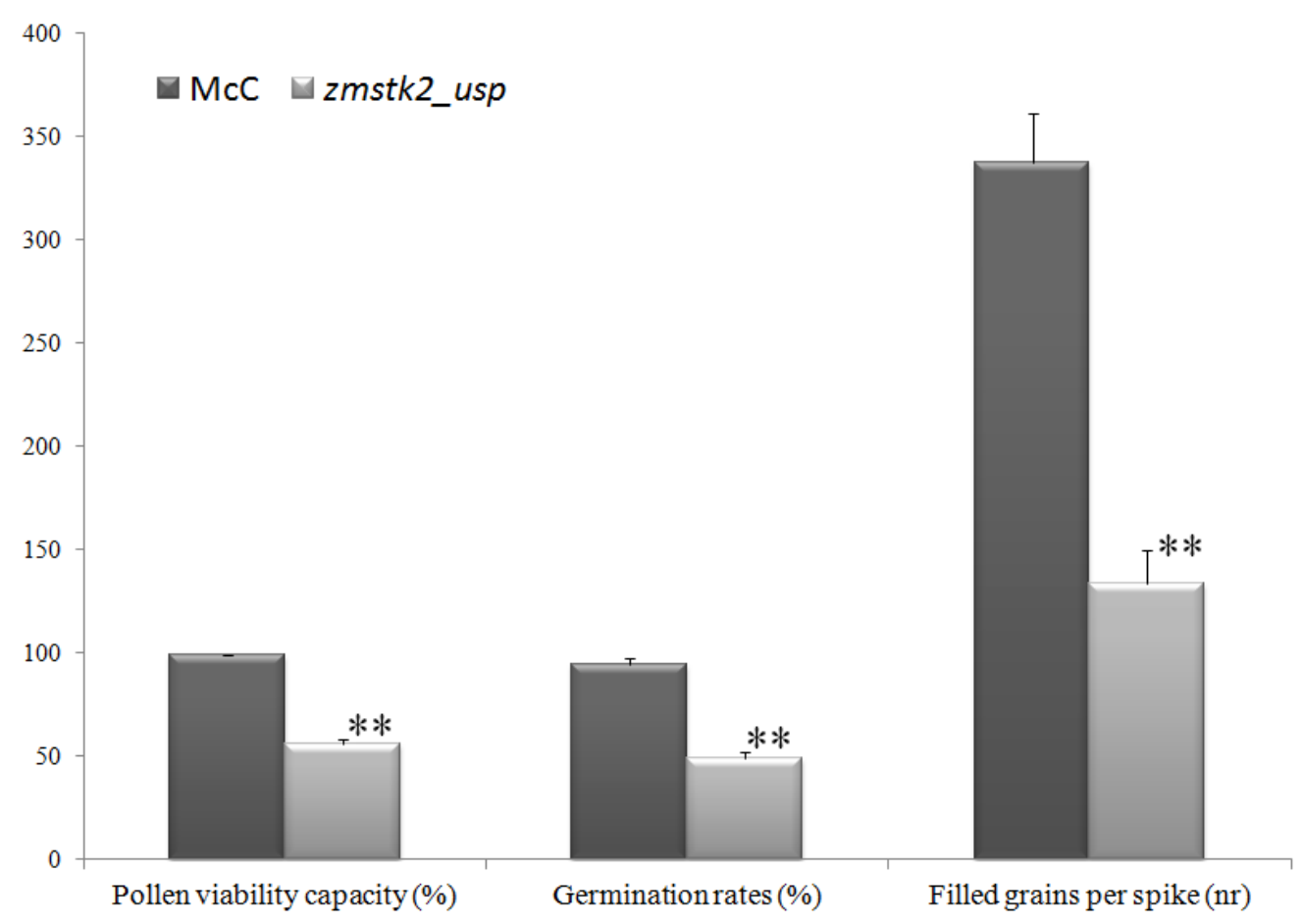

Figure 3. Analysis of pollen viability capacity (\%), germination rates (\%), filled grains per spike $(n r)$. Note: ** showed extremely significant difference. The error bars represent standard deviations of the means. Data mean $\pm S D(n=3)$

\section{Molecular biology detection of transgenic lines}

Using the method of Agrobacterium-mediated transformation of the maize germinating embryo (Wang et al., 2017), four positive plants were conformed by PCR and Southern blot (Fig. 4).

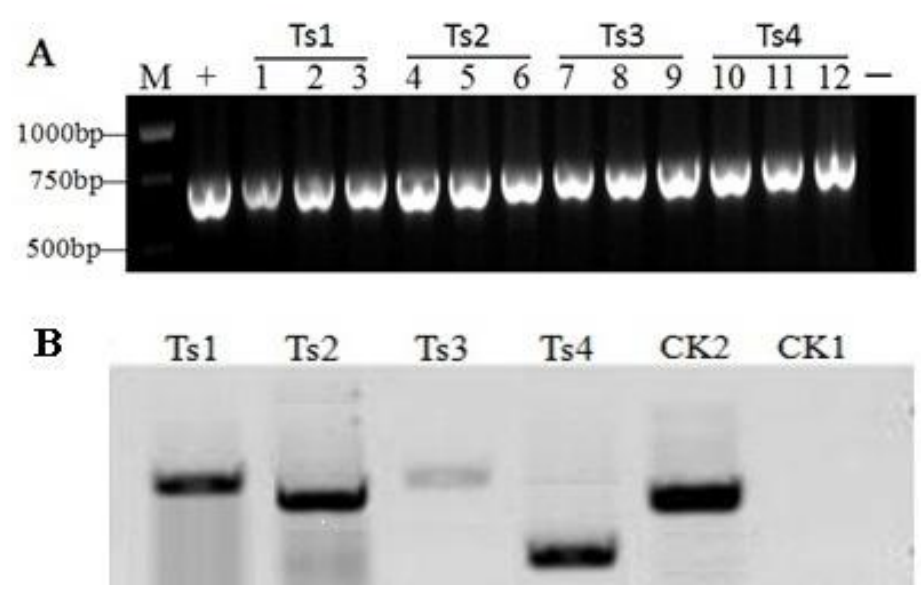

Figure 4. Molecular biology detection of transgenic lines. Note: A. PCR tests of ZmSTK2_USP-

GUS in $T_{0}$ transgenic lines; $B$. Southern blotting analysis of transgenic lines $\left(T_{0}\right) . M$ are

DL5000 and DL2000, respectively; + denotes transforming plasmids as positive control; denotes wild-type McC as negative control. Ts $1, T s 2, T s 3$ and Ts 4 are successfully transformed plants with ZmSTK2_USP-GUS. CK1 is the inbred line McC of non-genetically modified maize.

CK2 is the GUS gene 
Among the four positive transgenic lines with ZmSTK2_USP-GUS, only $\mathrm{T}_{2}$ seeds of Ts2 and Ts4 were obtained. To validate the tissue specificity of ZmSTK2_USP, using non-genetically modified maize $\mathrm{McC}$ as control, $\mathrm{T}_{2}$ seeds of Ts 2 and Ts4 expressing the GUS fusions, and non-genetically modified seeds (McC) were planted in a greenhouse, and the ZmSTK2_USP activity was examined by GUS staining at different developmental stages, then observed, photographed, and recorded under stereomicroscope. Ts2 and Ts4 showed no expression of GUS gene in immature male flower and early stage of pollens, while GUS activity was detected in middle stage, late stage of pollens and germinated pollen (Fig. 5). These results indicated that ZmSTK2_USP expressed at late stage of pollen development.

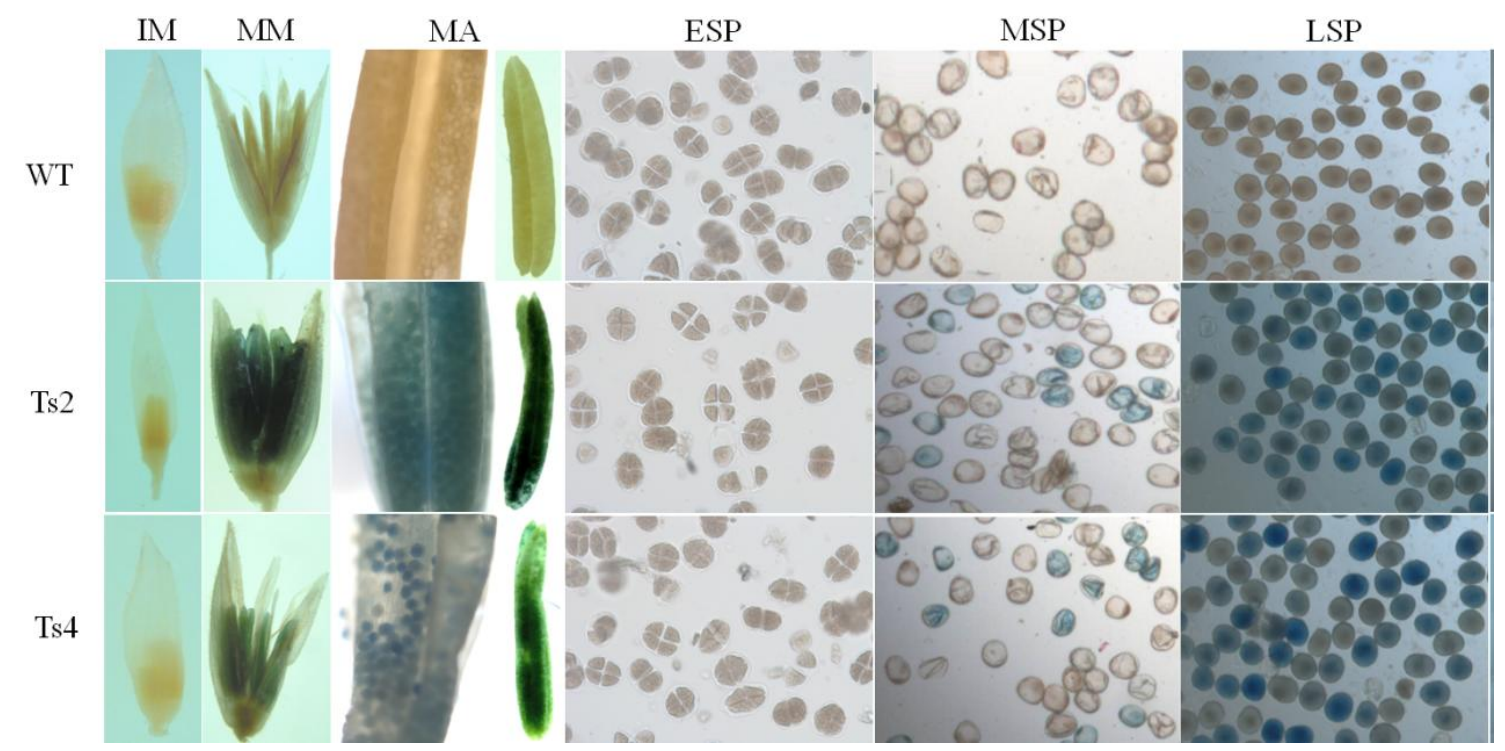

Figure 5. Histochemical GUS staining of maize transformed with ZmSTK2_USP-GUS. Note: WT is the inbred line McC of non-genetically modified maize; Ts 2 and Ts 4 are successfully transformed plants with ZmSTK2_USP-GUS; IM: Immature male flower; MM: Mature male flower; MA: Mature anther; ESP: early stage of pollens; MSP: middle stage of pollens; LSP: late stage of pollens

\section{Conclusion}

The molecular mechanisms involved in pollen development and pollen tube elongation are extremely complex. Besides a range of organ differentiation and tightly controlled changes in cellular physiology and biochemistry, a large number of genes are involved in expression and regulation. Most of these genes are also expressed in other tissues of plants, but there are still 10\%-20\% genes that are specifically expressed in pollen (Willing et al., 1988). At present, there are relatively few reports about pollen development genes in maize.

In our previous study, ZmSTK2_USP belong to the serine/threonine kinase (STK), containing a Usp domain on N-terminal (Wang et al., 2014; Zhou et al., 2014). In the present study, we cloned ZmSTK2_USP gene with specific-expressed at late stage of pollen development. The mutant ZmSTK2_USP, with wrinkled and withered pollen grains, showed $56.7 \%$ pollen viability capacity, $48.92 \%$ germination rates comparing with wild-type, which led to the reduction of seed setting rate. To investigate the specific expression of ZmSTK2_USP, Northern blot and qRT-PCR analysis results 
revealed that ZmSTK2_USP expressed at late stage of pollen development. Further, GUS staining of transgenic plants verified that ZmSTK2_USP gene has tissue specific expression at mature pollen. All these results are useful for providing theoretical basis for future study on the molecular mechanism of reproductive development in maize.

Acknowledgements. This research was supported by the National Natural Science Foundation of China (grant Nos. 31571672 and 3167101132).

\section{REFERENCES}

[1] Carpenter, J. L., Ploense, S. E., Snustad, D. P., Silflow, C. D. (1992): Preferential expression of an $\alpha$-tubulin gene of arabidopsis in pollen. - Plant Cell 4: 557-571.

[2] Fu, H., Park, W., Yan, X., Zheng, Z., Shen, B., Dooner, H. K. (2001): The highly recombinogenic bz locus lies in an unusually gene-rich region of the maize genome. Proc Natl Acad Sci USA 98: 8903-8908.

[3] Gagliardi, D., Breton, C., Chaboud, A., Vergne, P. Dumas, C. (1995): Expression of heat shock factor and heat shock protein 70 genes during maize pollen development. - Plant Molecular Biology 29: 841-856.

[4] Hanson, D. D., Hamilton, D. A., Travis, J. L., Bashe, D. M., Mascarenhas, J. P. (1989): Characterization of a pollen-specific cDNA clone from Zea mays and its expression. Plant Cell 1(2): 173-179.

[5] Jefferson, R. A., Kavanagh, T. A., Bevan, M. W. (1987): GUS fusions: ß-glucuronidase as a sensitive and versatile gene fusion marker in higher plants. - EMBO J 6(13): 39013907.

[6] Jin, Y. F., Bian, T. F. (2004): Isolation and expression of a wheat pollen-specific gene with long leader sequence. - J Integr Plant Biol 46(11): 1347-1356.

[7] Sambrook, J., Fritsch, E., Maniatis, T. (1989): Molecular Cloning: A Laboratory Manual, 2nd ed. - Cold Spring Harbor Laboratory Press: New York.

[8] Schreiber, D. N., Dresselhaus, T. (2003): In vitro pollen germination and transient transformation of Zea mays and other plant species. - Plant Molecular Biology Reporter 21: 31-41.

[9] Swapna, L., Khurana, R., Kumar, S. V., Tyagi, A. K., Rao, K. V. (2011): Pollen-specific expression of Oryza sativa indica pollen allergen gene (OSIPA) promoter in rice and Arabidopsis transgenic systems. - Molecular Biotechnology 48(1): 49-59.

[10] Wang, D. X., Wang, H., Irfan, M., Fan, M. X., Lin, F. (2014): Structure and evolution analysis of pollen receptor-like kinase in Zea mays and Arabidopsis thaliana. - Comput Bio Chem 51: 63-70.

[11] Wang, H., Mingxia, F., Wang, G., Zhang, C., Shi, L., Wei, Z., Ma, W., Chang, J., Huang, S., Lin, F. (2017): Isolation and characterization of a novel pollen-specific promoter in maize (Zea mays L.). - Genome 60(6): 485-495. dx.doi.org/10.1139/gen-2016-0089.

[12] Willing, R. P., Bashe, D., Mascarenhas, J. P. (1988): An analysis of the quantity and diversity of messenger RNAs from pollen and shoots of Zea mays. - Theor Appl Genet 75(5): 751-753.

[13] Zhou, P., Yang, F., Yu, J., Ao, G., Zhao, Q. (2010): Several cis-elements including a palindrome involved in pollen-specific activity of $S B g L R$ promoter. - Plant Cell Reports 29(5): 503-511.

[14] Zhou, T., Fan, M. X., Irfan, M., Wang, H., Wang, D. X., Wang, L., Zhang, C. Y., Lin, F. (2014): Phylogenetic analysis of STK gene family and Usp domain in maize. - Molecular Biology Reports 41: 8273-8284. 